\title{
Velhice e Saúde na Região da África Subsaariana: \\ uma agenda urgente para a cooperação internacional
}

\author{
Aging and health in Sub-Saharan Africa: \\ an urgent agenda for international cooperation
}

José Luiz Telles ${ }^{1}$

Ana Paula Abreu Borges ${ }^{1}$

\footnotetext{
${ }^{1}$ Departamento de

Administração e

Planejamento em Saúde,

Escola Nacional de Saúde

Pública, Fiocruz. Av. Brasil

4365, Manguinhos.

21.040-360 Rio de Janeiro

RJ Brasil.

jluiztelles@fiocruz.br
}

\begin{abstract}
The Sub-Saharan part of the African continent is the area that has the highest disease burden in the world and is the only region of the planet where it is expected that the number of poor people will increase in the coming decades. The countries of this region, to different degrees, experience slow process of population aging but at the same time, it is the are where the elderly population grows fastest in absolute numbers. Based on a review of the literature, an attempt was made to highlight the social and demographic situation in which the elderly live in the Sub-Saharan region and the main challenges faced by local governments to overcome the complex problems affecting society as a whole. It was found that public policies geared to this segment of the population in the region do not represent a priority and, consequently, are unlikely to be included in the current agenda of international cooperation.
\end{abstract}

Key words Population aging, Sub-Saharan Africa, Health and old age
Resumo A região Subsaariana do continente africano é onde se concentra a maior carga de doença do mundo e é a única região do planeta onde se espera que o número de pessoas pobres irá aumentar nas próximas décadas. Os países desta região, em diferentes graus, experimentam processo lento de envelhecimento populacional, mas, ao mesmo tempo, é onde a população idosa mais cresce em números absolutos. A partir de revisão da bibliografia, buscou-se destacar a situação demográfica e social em que vivem as pessoas idosas na região subsaariana e os principais desafios que se impõem aos governos locais para a superação dos complexos problemas postos a toda a sociedade. Constatou-se que as políticas públicas voltadas para este segmento populacional na região não representam prioridade e, por conseguinte, dificilmente entram na agenda atual da cooperação internacional.

Palavras-chave Envelhecimento populacional, África Subsaariana, Saúde e velhice 


\section{Introdução}

Envelhecimento populacional representa o aspecto dominante e mais visível da dinâmica da população mundial no século 21. Espera-se que no ano de 2050 as populações de numerosos países serão compostas com mais de $30 \%$ de pessoas acima de 60 anos. O Plano Madrid de Ação Internacional para o Envelhecimento, assinado pelos países membros das Nações Unidas no ano de 2002, fundamenta-se em três princípios básicos: a) participação ativa dos idosos na sociedade, no desenvolvimento e na luta contra a pobreza; b) fomento da saúde e bem-estar na velhice: promoção do envelhecimento saudável; e, c) criação de um entorno propício e favorável ao envelhecimento ${ }^{1}$.

Desde então, os países, em diferentes graus, têm buscado caminhos para que estes princípios se tornem realidade. Neste contexto, os estudos sobre o envelhecimento demográfico e suas consequencias para as políticas públicas cresceram significativamente, em particular nos países desenvolvidos. No entanto, a região da África Subsaarina talvez seja aquela em que menos se estruturam estudos e pesquisas sobre envelhecimento populacional $^{2}$.

Concomitante a esta realidade, na última década do século que se inicia, a saúde passa a ser questão que ultrapassa definitivamente as fronteiras nacionais. A saúde global, termo que busca dar sentido aos desafios de um mundo globalizado e em constantes mudanças, se caracterizaria "pelo sentido da responsabilidade coletiva pela saúde [a contemplar] os determinantes mais amplos da saúde a partir de uma perspectiva governamental integral e de envolver, de maneira tanto formal como informal, uma gama diversificada de interlocutores e interesses - articulando atores estatais e não estatais ${ }^{3}$. No cenário da saúde global, a cooperação entre os países em diversas áreas, incluso a da saúde, tem se intensificado e adquirindo novos contornos. A cooperação entre países do Hemisfério Sul, ou simplesmente, cooperação sul-sul, ganha relevância na medida em que pode ser identificada como um tipo de cooperação horizontal, "objetivando-se uma concertação com vistas à obstrução das desigualdades internacionais dirigidas pelos países desenvolvidos do Norte, bem como ações conjuntas com vistas ao enfrentamento de problemas domésticos semelhantes" ${ }^{4}$. O envelhecimento populacional é uma das questões que gradativamente ganha terreno no âmbito das cooperações internacionais. Mas até onde as consequencias do envelhecimento demográfico em África tem sido foco de atenção dos governos locais? Não o sendo, conforme deveremos explicitar neste artigo, dificilmente será tema da agenda de desenvolvimento de acordos de cooperação sul-sul.

O objetivo desta revisão é caracterizar demografica e socialmente o processo de envelhecimento no continente africano, com foco na África Subsaariana, com o intuito de trazer à luz questões essenciais para a agenda da saúde global tendo por referência os princípios básicos do Plano Internacional para o Envelhecimento.

A pesquisa bibliográfica teve por referência o Portal PubMed na base de artigos Medline ao longo de 2011. A finalidade foi a de levantar a literatura publicada com descritores relacionados com envelhecimento populacional, doenças cronicas não transmissíveis, saúde do idoso, condições de saúde e de vida em África Subaariana.

A estratégia genérica de busca foi: "Health of the Elderly" OR "Demographic Aging” OR “Chronic Disease" OR "Health Status" OR "Social Conditions" AND "Africa”.

Outras bases de dados como Scielo, Lilacs, Google e Google Acadêmico foram visitadas a partir de artigos de referência previamente selecionados, o que possibilitou acesso à literatura não indexada ao Medline. Outras fontes foram importantes, tais como documentos e relatórios técnicos, para complementar a pesquisa.

\section{Transição demográfica e envelhecimento populacional}

A transição demográfica é o processo pelo qual um país passa de um regime com alta mortalidade e alta fecundidade para outro caracterizado por baixa mortalidade e baixa fertilidade ${ }^{5}$.

Nos anos 1960 foi observado um declínio na mortalidade em quase todos os países em desenvolvimento. Entretanto, as taxas de fertilidade ainda estavam elevadas. O resultado imediato foi o rápido crescimento populacional nestes países o que fez alguns estudiosos afirmarem que o mundo estava caminhando para uma catástrofe. Nas décadas posteriores, entretanto, o que se viu foi um rápido decréscimo nas taxas de fertilidade nas regiões da Ásia, América Latina e Norte da África ${ }^{6}$.

$\mathrm{Na}$ África Subsaariana também tem sido observado o processo de transição demográfica. A falta de dados, no entanto, dificulta precisar quando as taxas de mortalidade começaram a declinar. Os dados disponíveis apontam que a queda se deu no início dos anos 1950. A tendência de 
queda nas taxas de mortalidade continuou nas décadas seguintes e, com taxas de fertilidade relativamente estáveis, o resultado desta equação foi maior aceleração do crescimento geral da população $0^{6-9}$.

Durante pelo menos três décadas, o declínio das taxas brutas de mortalidade e, também, das taxas do componente infantil (óbitos menores de um ano) manteve-se relativamente uniforme em todas as regiões da África Subsaariana. A partir dos anos 1990, em função da emergência da epidemia de HIV/AIDS em fins dos anos 1980, verifica-se piora nos indicadores de mortalidade em toda a região.

Com relação à fertilidade, a média geral na região é significativamente alta para os atuais padrões internacionais, em especial se comparadas com os países em desenvolvimento, tanto da América Latina quanto do continente asiático. Alguns fatores são relacionados à alta taxa de fertilidade observada na maioria dos países da África Subsaariana: taxas elevadas de mortalidade infantil ${ }^{6,10,11}$; casamentos e gestações preco$\operatorname{ces}^{10}$; baixa utilização de métodos contracepti$\operatorname{vos}^{10,11}$; valorização social da mulher com prole numerosa ${ }^{10,12}$; baixa escolaridade das mulheres ${ }^{11}$; a prática de poligamia, muito comum principalmente nas áreas rurais ${ }^{10}$; baixo desenvolvimento economico ${ }^{6,11}$ e garantia de sobrevivência - "proteção social demográfica" ${ }^{12}$. Há consenso nos estudos que todos os fatores relacionados às altas de fertilidade estão direta ou indiretamente interligados e alguns são interdependentes.

Para completar a caracterização da transição demográfica na região subsaariana, observa-se que a tendencia de aumento gradativo na esperança de vida ao nascer verificada tanto nos países desenvolvidos quanto naqueles em desenvolvimento não ocorreu em alguns países da região subsaariana. Em alguns deles verificou-se mesmo um decréscimo na expectativa de vida. Este fenômeno é em larga medida decorrência do impacto da epidemia de HIV/AIDS no continente africano em geral e nos países da região subsaariana em particular. É certo que outras situações concorreram para a estagnação ou diminuição da longevidade tais como os conflitos violentos, que ainda levam, nos dias de hoje, milhares de pessoas a se refugiarem em outros países; a escassez de recursos de atenção à saúde e as péssimas condições gerais de vida. Entretanto, em muitos países, é o HIV/Aids a principal razão para tal fenômeno ${ }^{7}$.

A tendência da expectativa de vida ao nascer no Zimbabwe é um bom exemplo para ilustrar a devastação causada pela epidemia de HIV/Aids. A expectativa ao nascer caiu de 61.6 anos em 1986 para 40.4 anos em 2006, isto é, um decréscimo de 21 anos. $\mathrm{O}$ impacto para as mulheres foi ainda maior. A expectativa de vida para o sexo feminino neste país no mesmo período decresceu 27 anos. Tendencias similares são observadas em outros países da região que tiveram sua população afetada pelo HIV/Aids ${ }^{7}$.

O fato de os países que compõem a região subsaariana da África terem as menores expectativas de vida ao nascer do mundo não quer dizer, em absoluto, que não existam pessoas idosas nesta área do planeta. É verdade que a África Subsaariana, em contraste com as outras regiões, é a que tem menor proporção de população idosa e, também, menor crescimento relativo.

A projeção da média de proporção de pessoas idosas por região da África Subsaariana no ano de 2011 está assim distribuída: a) África Ocidental - 4,2\%; b) África Central - 4,5\%; c) África Austral - 8,3\%; e d) África Oriental - 4,7\% ${ }^{13}$.

As projeções futuras mantêm a tendencia de a Região Subsaariana possuir a menor proporção de pessoas idosas entre suas populações. Enquanto que na Europa, em 2030, projeta-se uma proporção de $28 \%$ de pessoas com 60 anos e mais, na África Subsaariana esta proporção será de $5,6 \%{ }^{7}$.

Entretanto, quando se analisa a população idosa em números absolutos o cenário se apresenta bastante diferente. As atuais projeções demográficas mostram que a região é onde se verifica o maior crescimento populacional de pessoas idosas no mundo. Em termos absolutos, se verificará um crescimento significativo deste contingente populacional nesta região passando de 42,6 milhões em 2010 para 160 milhões no ano de $2050^{13}$.

As pessoas da região que conseguem alcançar os 60 anos de idade podem esperar viver mais anos quase tanto quanto as pessoas da mesma idade em outras regiões do mundo ${ }^{14}$.

Os números impressionam ainda mais quando se correlaciona o aumento expressivo da população idosa na África Subsaariana ao atual nível de desenvolvimento econômico e social e, ain$\mathrm{da}$, às transformações pelas quais a maioria dos países desta região está a apresentar.

\section{Envelhecimento e Pobreza}

A saúde de uma determinada população é afetada ao longo da vida pelas características do contexto social, que geram desigualdades nas 
exposições e vulnerabilidades. Esses determinantes sociais interferem no bem-estar, independência funcional e qualidade de vida da população idosa $^{15}$. Apesar das limitações em considerar as medidas de pobreza convencionais (ex. linhas de pobreza) como indicadoras fiéis da real condição de vida ${ }^{16}$, principalmente dos idosos, o grau de pobreza pode expressar os limites aos quais a população idosa pode estar sujeita.

A região subsaariana da África é a mais pobre região do mundo, o que implica que o envelhecimento da sua população é em grande parte vivenciado em contextos de pobreza generalizada e de profunda restrição economica. Entre os dez países que possuem o menor rendimento nacional bruto do mundo, nove estão nesta região ${ }^{17}$.

A África Subsaariana terminou o último milênio mais pobre do que era em 1990. Em 2005, 23 países da região eram mais pobres do que eram em 1975. Neste mesmo ano de 2005, mais de $50 \%$ dos habitantes desta região viviam com menos de USD 1,00 por dia, a grande maioria da população abaixo de USD 2,00 por dia e, globalmente, é a única região onde se espera que a proporção de pessoas vivendo na pobreza cresça ${ }^{14}$.

Apesar de atingir a população em todas as faixas etárias, as crianças e as pessoas idosas são mais vulneráveis à pobreza. A incidencia de pobreza agrava-se de acordo com os arranjos familiares. Residencias onde há algum morador idoso, ou onde há pessoas idosas a coabitarem com crianças menores de 10 anos e domicílios chefiados por pessoas idosas estão, em geral, mais sujeitos à incidencia de pobreza se comparado com a média geral na população. Nos países onde a incidência do HIV-AIDS é muito alta, as diferenças são maiores e estatisticamente significativas ${ }^{18}$.

Uma das medidas de redução da pobreza na população idosa tem sido a adoção de pensões não contributivas para aquelas parcelas mais pobres. Evidências sugerem que a pobreza entre os idosos é baixa em países onde existem mecanismos de transferência de renda dirigida para esta parcela da população, como no Brasil, Chile ou África do Sul. Em contraste, em países onde os sistemas de pensões na velhice são inexistentes ou atingem um número reduzido de pessoas, as mais velhas estão sobrerrepresentadas entre os pobres $^{19,20}$. Além disso, nos países em desenvolvimento os efeitos positivos das pensões vão além dos beneficiários diretos (os idosos) e refletemse para os outros membros de suas famílias. Estudos de caso no Brasil e África do Sul mostram que as crianças dentro de famílias beneficiárias têm maiores taxas de escolarização e melhor es- tado de saúde do que aqueles que vivem em domicílios que não recebem uma pensão ${ }^{21}$.

No entanto, apesar de um papel comprovadamente positivo, a maioria das populações do continente africano permanece descoberta por um regime de pensões. Exceto em alguns países (África do Sul, Namíbia, Ilhas Maurícios e Botswana), quase nenhum país africano tem colocado ênfase na ampliação da cobertura dos sistemas de pensões, quando estes existem, ou na criação de um programa consistente de proteção social para a população idosa ${ }^{2}$.

Tal posição pode ser explicada pela concordância da maioria dos países do continente ter assumido as recomendações do Banco Mundial no seu relatório sobre a crise de velhice divulgado em 199422. O argumento, então, do Banco Mundial era de que os sistemas tradicionais de apoio para os idosos nas sociedades africanas, pricipalmente tendo por base a família, estão trabalhando muito bem e que os regimes de pensões formais iriam desestimular as transferências familiares, agravando ainda mais as condições de convivência para os idosos. Devido a essa recomendação, a questão da provisão de pensões tem sido raramente considerada em programas de desenvolvimento e em estratégias de redução da pobreza na África ${ }^{2}$.

$\mathrm{O}$ argumento de que as famílias tradicionais na região estão a prover os cuidados necessários às pessoas idosas não leva em consideração as transformações sociais que estão a ocorrer na maioria dos países da África Subsaariana.

O crescimento bruto da população nesta região, tanto na área rural (aumento anual de $1,9 \%$ ) quanto na urbana (aumento anual de 4\%) é a mais alta do mundo. Desde 1990 sua população urbana vem aumentando até ter dobrado para 290 milhões de pessoas em 2007. Embora a África Subsariana continue a ser predominantemente rural, verifica-se rápida urbanização. Em 1990, 28\% da população vivia em zonas urbanas. No ano de 2007, este percentual subiu para $36 \%$. Tal crescimento pressionou significativamente a demanda por serviços urbanos. Por exemplo, no ano de 2006 , somente $57 \%$ da população urbana e 23\% da área rural tinha acesso a instalações sanitárias melhoradas ${ }^{23}$.

$\mathrm{Na}$ medida em que as sociedades africanas, tradicionalmente caracterizadas por economias baseadas primordialmente em agricultura familiar, movem-se para um estilo individualizado e urbanizado de vida, o status tradicional das pessoas idosas torna-se ameaçado e seus papéis na família e na comunidade transformados. A ur- 
banização altera valores, atitudes e padrões individuais e, por conseguinte, os próprios regimes culturais. Inicia-se com a repartição mais ou menos acentuada e acelerada da ordem social tradicional e termina por minar a capacidade tradicional de apoiar, integrar e dar sentido à vida nas faixas etárias mais avançadas, onde se verificam, com frequencia, incapacidades ${ }^{24}$.

Além do processo de urbanização, a epidemia de HIV/Aids vem desempenhando papel crucial para as mudanças verificadas nos arranjos familiares desta região.

Enquanto os dados de 2007 da UNAIDS mostravam que a epidemia de Aids parecia ter atingido seu pico e as taxas de mortalidade estavam a cair, mais de dois terços de todas as pessoas que vivem com HIV residem na África Subsaariana, onde ocorreram mais de três quartos (76\%) de todas as mortes por Aids em 2007. Estima-se que 22,5 milhões de africanos vivam com HIV/AIDS, a grande maioria deles adultos no auge da vida profissional ${ }^{25}$.

A epidemia de HIV/Aids neste continente está a esgarçar o próprio tecido da vida cotidiana com profundas implicações para o desenvolvimento social e econômico das gerações seguintes.

Primeiro, porque a epidemia atinge principalmente as pessoas em plena capacidade laboral. As roças familiares, em particular, são negligenciadas ou abandonadas por motivos de doença em algum membro da família, contribuindo ainda mais para a insegurança alimentar em muitas áreas. Em países de alta prevalência, existe um ciclo vicioso entre a escassez de alimentos, desnutrição e $\mathrm{AIDS}^{26}$. No Zimbábue, a prevalência do HIV em pessoas adultas é de cerca de $25 \%$. Em 2000, a Aids havia ceifado o país entre 5\% a $10 \%$ de sua força de trabalho agrícola. No Malawi, as famílias que perderam as mulheres com idade inferior a 60 anos tiveram duas vezes mais probabilidade de sofrer um déficit alimentar do que em famílias nas quais os homens na mesma faixa etária morreram ${ }^{27}$.

Em segundo lugar, a AIDS enfraquece ou até mesmo destrói os mecanismos que geram a formação de capital humano ${ }^{28}$.

Por último, a epidemia deixou, ao longo das ultimas três décadas, milhares de crianças órfãs pelo continente. Estimativas apontam que cerca de 12 milhões de crianças entre 0-17 anos perderam um ou ambos os pais devido à Aids na região da África Subsaariana. Como resultado, o número total de crianças órfãs por todas as causas na região está em expansão e atingiu 48,3 milhões no final de $2005^{29}$.
Análise de dados em 24 países da África Subsariana constatou forte associação entre a mortalidade relacionada à Aids dentro de um país e a probabilidade de encontrar uma pessoa idosa cuidando de uma ou mais crianças órfã s ${ }^{30}$.

O cuidado de crianças órfãs por pessoas idosas em geral é precedido do cuidado dos pais das crianças. Estudo da Organização Mundial de Saúde sobre cuidadores(as) idosos(as) no Zimbabwe constatou que 39\% afirmou ter experimentado doença física após a morte de um parente por HIV/Aids que estava sob seus cuidados e número quase igual relatou estresse emocional ${ }^{31}$.

Apesar de ser, muitas das vezes, experienciado como sobrecarga no dia a dia, a percepção do cuidado por parte das pessoas idosas pode ter outro significado. Em estudo realizado em área rural da África do Sul com mulheres idosas cuidadoras, houve hesitação por parte das entrevistadas ao relacionar os cuidados sob sua responsabilidade como sendo uma "carga" na medida em que estavam simplesmente "cuidando de seu próprio sangue" 32 .

A gravidade da epidemia do HIV/Aids, ain$\mathrm{da}$, foi significativa e positivamente associada com aumento da proporção de pessoas idosas vivendo sozinhas ${ }^{33}$.

\section{Envelhecimento Demográfico e Carga de Doenças}

A transição demográfica é associada à epidemiológica, isto é, mudanças ocorridas ao longo de um determinado tempo nos padrões de morte, morbidade e invalidez que caracterizam uma população específica e que, em geral, ocorrem concomitante a outras transformações ${ }^{34}$. A transição epidemiológica engloba três mudanças básicas no padrão de mortalidade de uma dada sociedade: a) substituição das doenças transmissíveis por outras não transmissíveis e causas externas; b) deslocamento da carga de morbi-mortalidade dos grupos mais jovens aos mais idosos; e, c) transformação de uma situação em que predomina a mortalidade para outra na qual a morbidade é dominante ${ }^{35}$.

$\mathrm{Na}$ África Subsaariana, os dados que permitem caracterizar a passagem de um estágio da transição para o próximo são escassos. Entretanto, existem evidências demonstrando que o perfil das doenças vem experimentando mudanças importantes em um contexto cada vez marcado pela coexistência de doenças infecciosas, de deficiências nutricionais e de doenças crônicas não transmissíveis. Os dados disponíveis apontam 
que a crescente urbanização está a contribuir para estilos de vida pouco saudáveis (mudanças nos hábitos alimentares, sedentarismo, tabagismo e maior consumo de álcool) levando ao aumento dos riscos para as doenças coronarianas ${ }^{36}$.

Os Estudos sobre a Carga Global de Doença de 1990 e de 2000 estimam que a África Subsaariana tem a maior carga de doença no mundo ${ }^{37}$. A região tem registrado aumento acelerado das doenças não transmissíveis (DNT), incluindo violência e lesões, aumentando a carga já pesada das doenças transmissíveis. Projeta-se, se não houver mudanças significativas em relação ao cuidado e à prevenção, que as doenças não transmissíveis representarão pelo menos $50 \%$ da mortalidade no continente africano em 2020. Os principais fatores de risco para as doenças crônicas não transmissíveis estão relacionados com estilos de vida individuais e fatores de risco não modificáveis, incluindo considerações genéticas e étnicas, juntamente com o aumento gradativo da expectativa de vida, fatores pré-natais e de gênero ${ }^{38}$.

As doenças cardiovasculares são as causas secundárias mais comuns de óbitos de adultos na África Subsaariana, além de ser uma das principais causas de doença crônica e de incapacidade nas idades mais avançadas. Metade das mortes por doenças cardiovasculares ocorre em pessoas na faixa etária entre os 30 e os 69 anos de idade. Comparada com as regiões mais desenvolvidas, morre-se na África Subsaariana 10 ou mais anos mais jovem por doenças cardiovasculares ${ }^{39}$.

Uma proporção considerável de pessoas idosas sofre de desnutriçãa ${ }^{40}$, de múltiplas condições crônicas físicas e mentais (tais como as condições músculo-esquelético e cardiovascular, deficiências visuais ou auditivas, depressão e demência) ${ }^{41-44}$ e, por consequencia, possuem uma saúde bastante debilitada ${ }^{45,46}$.

Ademais, adultos e pessoas mais velhas portadoras de doenças cronicas não transmissíveis, muitas vezes, não têm acesso a serviços necessários para procedimentos de diagnóstico precoce, de tratamento adequado e de prevenção de outros agravos e complicações $s^{47,48}$.

\section{O Envelhecimento Demográfico \\ e Agenda dos Governos de África}

No ano de 2003, como desdobramento do Plano de Ação Internacional sobre o Envelhecimento das Nações Unidas de 2002 (Plano de Madrid), a União Africana publicou o documento intitulado Plano de Ação sobre o Envelhecimento da União Africana ${ }^{49}$. Neste documento propugna-se o desenvolvimento de estratégias para melhorar a prestação de serviços de saúde para pessoas idosas da África como uma maneira de realizar seu direito à saúde e de promover a sua contribuição para as famílias e as sociedades. Ambos os planos atentam para dois tipos principais de medidas: (i) estratégias multifacetadas de promoção da saúde para prevenir doenças e invalidez entre os distintos grupos etários que compõe a velhice; e, (ii) políticas específicas para assegurar o pleno acesso aos cuidados curativos e de reabilitação adequados para pessoas mais velhas doentes e/ou portadores de algum grau de deficiência/incapacidade ${ }^{50}$.

Entretanto, desde a publicação do Plano, o cenário da atenção à saúde da população idosa na África Subsaariana pouco mudou. Parece haver falta de convicção de que a ação sobre a saúde da população idosa deva ser uma prioridade dentro de um contexto de alta prevalência de doenças infecto-contagiosas com impacto direto na morbimortalidade materno infantil ${ }^{50}$.

A postura aparentemente típica dos legisladores e dos profissionais de planejamento nos níveis centrais de governo, em especial aqueles relacionados às finanças do país, é que os orçamentos nacionais não têm capacidade de sustentar políticas específicas para o segmento da população idosa em seus países. Tais políticas, ao contrário de programas essenciais de saúde voltados para crianças, jovens e adolescentes são vistas como um obstáculo, ou na melhor das hipóteses, como irrelevantes para os interesses de desenvolvimento do país. Ou, ainda, há falta de clareza sobre quais as medidas específicas necessárias para efetivamente garantir a saúde das pessoas idosas ${ }^{48}$. Junte-se a isso, todas as carências cronicas, materiais e humanas dos serviços de saúde nesta região.

\section{Considerações Finais}

O processo de envelhecimento demográfico verificado na grande maioria dos países que pertence à região da África Subsaariaana dá-se de forma lenta em virtude das altas taxas de fertilidade ainda presentes nesta região. No entanto, o número de pessoas idosas está a crescer de forma intensa em toda a região. As doenças infectocontagiosas ainda são responsáveis por grande parte da mortalidade geral, com destaque importante para a epidemida de HIV/AIDS que tem reconfigurado as estruturas familiares. Ademais, o processo de urbanização e as mudanças de estilos de vida têm 
contribuido para o aumento das doenças cronicas não transmissíveis. Assim, configura-se um quadro de dupla carga de doença que traz sérios desafios para a organização e para o financiamento dos sistemas nacionais de saúde.

Neste contexto, o acesso ao diagnóstico, tratamento e ações de prevenção por parte da população idosa é precária. Por outro lado, os governantes, apesar de instituirem documentos que firmam compromisso com a melhoria da qualidade de vida deste segmento populacional, ainda não incorporaram efetivamente nas agendas das políticas públicas as necessidades e as demandas da população idosa.

A concertação em torno dos objetivos e metas do milênio, instituído pelas Nações Unidas no ano de 2000, tornou-se referência central para as iniciativas de cooperação internacional que têm como foco os países em desenvolvimento. Ainda que possam ter relação com as condições de vida da população idosa em geral, a agenda específica do envelhecimento populacional e suas consequencias acabam subsumidas por outras prioridades, notadamente a redução da mortalidade infantil e materna. Tal fato terá, a médio e longo prazo, implicações profundas na capacidade dos governos que compõem a região subsaariana em enfrentar os desafios impostos pelo envelhecimento demográfico em seus países. Trata-se, portanto, de uma agenda que deveria ser o mais rapidamente incluída nos termos das cooperações entre os países, em especial os do hemisfério sul-sul.

\section{Colaboradores}

JL Telles participou da escolha dos temas, do levantamento bibliográfico e da redação e revisão final do texto. APA Borges participou na organização da bibliografia de acordo com os temas e redação do texto. 


\section{Referências}

1. Camarano AA, Pasinato MT. O Envelhecimento Populacional na Agendas das Políticas Públicas. In: Camarano AA, organizador. Os Novos Idosos Brasileiros: Muito Além dos 60? Rio de Janeiro: IPEA; 2004. p. 253-292.

2. Faye O. Basic Pensions and Poverty Reduction in sub-Saharan Africa. The WDA-HSG Discussion Paper Series on Demographic Issues: No. 2010/2. University of St. Gallen. 2010. 22p. [cited 2011 Aug 26]. Disponível em: http://www2.ulg.ac.be/crepp/papers/ crepp-wp200707.pdf.

3. Kickbusch I, Berger C. Diplomacia da Saúde Global. RECIIS - R. Eletr. de Com. Inf. Inov. Saúde [periódico na Internet]. 2010 Mar. [acessado 2011 out 18]; 4(1):[19-24]. Disponível em: http://www. reciis.cict.fiocruz.br/index.php/reciis/article/view/ 342

4. Rodrigues DF. Cooperação horizontal Sul-Sul: arranjos de concertação política entre a Índia, o Brasil e a África do Sul. Rev. bras. polit. int. [periódico na Internet]. 2010, [acessado 2011 Out 26]; 53(1):[4566]. Disponível em: http://www.scielo.br/scielo. php?script=sci_arttext\&pid $=$ S0034-73292010000100 $003 \& \operatorname{lng}=$ pt\&nrm $=$ iso.

5. Prata PR. A Transição Epidemiológica no Brasil. Cad Saude Publica 1992; 8(2):168-175.

6. Malmberg B. Demography and the Development Potential of sub-Saharan Africa. Current African Issues 38. [serial on the Internet] 2008 [cited 2011 Aug 31]. Available from: http://www.regeringen.se/ $\mathrm{sb} / \mathrm{d} / 9037 / \mathrm{a} / 85210$.

7. Velkoff VA, Kowall PR. Population Aging in SubSaharan Africa: demographic dimensions - 2006. US Census Bureau, Current Population Reports P95/ 07-1. [document on the Internet] 2007 Jun [cited 2011 Aug 23] [about 39p]. Available from: http:// www.census.gov/prod/2007pubs/p95-07-1.pdf

8. Caldwell JC, Caldwell P. The Fertility Transition in Sub-Saharan Africa. Conference Fertility and the Current South African Issues of Poverty, HIV/AIDS and Youth. Pretoria: [document on the Internet] 2002 Oct [cited 2011 Aug 29]. Available from: http:// www.sarpn.org/documents/d0000082/P79_Caldwell. pdf.

9. United Nations (UN). World Population Prospects: the 2008 revision - Highlights. [document on the Internet] 2009 [cited 2011 Sep 03]. Available from: http://www.un.org/esa/population/publications/ wpp2008/wpp2008_highlights.pdf.

10. Bigombe B, Khadiagala GM. Major trends affecting families in sub-Saharan Africa. In: United Nations. Major Trends Affecting Families: A Background Document, Report for United Nations, Department of Economic and Social Affairs, Division for Social Policy and Development, Program on the Family. [document on the Internet] 2003 May. [cited 2011 Sep 05]. Available from: http://social.un.org/index/Link Click.aspx?fileticket $=3 \mathrm{VvV} \_\mathrm{mL} 8 \mathrm{oMw} \% 3 \mathrm{~d}$ \&tabid $=282$

11. Kreider A, Shapiro D, Varner C, Sinha M. Socioeconomic Progress and Fertility Transition in the Developing World: Evidence from Demographic and Health Surveys. [document on the Internet] 2009 Dec. [cited 2011 Aug 30]. Available from http://econ.la. psu.edu/papers/IUSSP2009FertilityPaper-20Dec.pdf.
12. Francisco AAS. A Natureza Incipiente da Transição Demográfica em Moçambique. Revista de Estudos Demogáficos. [periódico na Internet], 2011 [acessado 2011 ago 15]; 49:[5-36], Disponível: http://www. ine.pt/xportal/xmain? xpid=INE\&xpgid=ine publicacoes\&PUBLICACOESpub_boui $=121044272$ $\&$ PUBLICACOESmodo $=2$.

13. U.S. Census Bureau, International Data Base. [document on the Internet] 2011. [cited 2011 Sep 04]. Available from: http://www.census.gov/population/ international/data/idb/region.php? $\mathrm{N}=\% 20$ Region $\%$ 20Results $\% 20 \& \mathrm{~T}=2 \& \mathrm{~A}=$ aggregate $\& \mathrm{RT}=0 \& \mathrm{Y}=2011$ $\& \mathrm{R}=111,112,114,115 \& \mathrm{C}=$

14. Aboderin I. Understanding and Responding to Ageing, Health, Poverty and Social Change in SubSaharian Africa: a strategic framework and plan for research. Outcomes of the Oxford Conference on Research on Ageing, Health and Poverty in Africa: forging directions for the future. [document on the Internet] 2005 May. [cited 2011 Aug 18]. Available from: http://www.ageing.ox.ac.uk/files/afran_oxford_ conf_research_ageing_africa_final_report.pdf

15. Geib, LTC. Determinantes sociais da saúde do idoso. Cienc Saude Colet 2012; 17(1):123-133.

16. Bento JA, Lebrão ML. Suficiência de renda percebida por pessoas idosas no Município de São Paulo/Brasil. Cienc Saude Colet 2013; 18(8):2229-2238.

17. World Bank. Atlas Global of Development: A visual guide to the world's greatest challenges. $3^{\text {th }}$ Edition. Washington DC: The World Bank, Collins; 2011.

18. Kakwani N, Subbarao K. Aging and Poverty in Africa and the Role of Social Pensions. Social Protection Unit/Human Development Network/The World Bank. [document on the Internet] 2005 Jun. [cited 2011 Sep 10]. Available from: Disponível: http://site resources.worldbank.org/SOCIALPROTECTION/ Resources/SP-Discussion-papers/Safety-Nets-DP/ 0521.pdf

19. Barrientos A. "What is the impact of non-contributory pensions on poverty? Estimates from Brazil and South Africa", Working Paper IDPM/Chronic Poverty Research Centre. [document on the Internet] 2003. [cited $2011 \mathrm{Sep}$ 03]. Available from: http:/ /www.chronicpoverty.org/uploads/publication files/WP33_Barrientos.pdf

20. Barrientos A, Gorman M, Heslop A. (2003). “Old age poverty in developing countries contributions and dependence in later life", World Development. [serial on the Internet], 2003 [cited 2011 Sep 03]; 31(3):[555-570]. Available from: http://www.science direct.com/science/article/B6VC6-480CHY3-8/2/ 83967ele362f64f51ba74a6fc1082c53

21. Duflo E. "Grandmothers and granddaughters: old age pension and intrahousehold allocation in South Africa", National Bureau of Economic Research Working Paper 8061 [document on the Internet], 2000; [cited 2011 Aug 26]; [about 36p]. Available from: http://www.nber.org/papers/w8061

22. World Bank. Averting the Old Age Crisis: Policies to Protect the Old and Promote Growth [document on the Internet]. 1994 [cited 2011 Sep 10]. Available from: http://www-wds.worldbank.org/external/default/ WDSContentServer/WDSP/IB/1994/09/01/00000 9265_3970311123336/Rendered/PDF/multi_page.pdf 
23. World Bank. Regional Fact Sheet From The World Development Indicators [document on the Internet]. 2009 [cited 2011 Sep 4]. Available from: http:// siteresources.worldbank.org/DATASTATISTICS/ Resources/ssa_wdi.pdf

24. Apt NA. Ageing in Africa. Paper prepared for World Congress on Medicine and Health [document on the Internet]. 2000 July - Aug. [cited 2011 Sep 4]. Available from: http://www99.mh-hannover.de/aktuelles/projekte/mmm/englishversion/fs_programme/ speech/Apt_V.html

25. Joint United Nations Programme on HIV/AIDS (UNAIDS). AIDS Epidemic Update [document on the Internet]. 2007. [cited 2011 Sep 4]. Available from: http://data.unaids.org/pub/EPISlides/2007/ 2007_epiupdate_en.pdf.

26. Food and Agriculture Organization (2001). The impact of HIV/AIDS on food security. Committee on World Food Security, Twenty-seventh Session [document on the Internet]. 2001 May-Jun. [cited 2011 Sep 4]. Available from: http://www.fao.org/docrep/ meeting/003/Y0310E.htm.

27. Southern Africa Development Community - Food, Agriculture and Natural Resources Vulnerability Assessment Committee Towards identifying impacts of HIV/AIDS on food security in Southern Africa and implications for response: findings from Malawi, Zambia and Zimbabwe [document on the Internet]. 2003 [cited 2011 Sep 4]. Available from: http://www. sadc. int/fanr/aims/rvaa/Documents/VAA_Dissemination $12003 / 2003 \% 20$ Impact $\% 20$ of $\% 20$ HIV-AIDS $\%$ 20on\%20Food\%20Security.pdf

28. Fortson J G. Mortality Risk and Human Capital Investment: the impact of HIV/AIDS in Sub-Saharan Africa. The Review of Economics and Statistics [serial on the Internet] 2011 [cited 2011 Sep 5]; 93(1):[1-15]. Available from: http://www.mitpress journals.org/doi/pdf/10.1162/REST_a_00067

29. The United Nations Children's Fund (UNICEF). Africa's Orphaned and Vulnerable Generations: Children Affected by Aids [document on the Internet]. 2006 Aug. [cited 2011 Sep 5]. Available from: http:/ /www.unicef.org/publications/files/Africas_ Orphaned_and_Vulnerable_Generations_Children_ Affected_by_AIDS.pdf

30. Zimmer Z, Dayton J. The Living Arrangements of Older Adults in Sub-Saharan Africa in a Time of HIV/AIDS. Population Council: Policy Research Division [document on the Internet]. 2003 [cited 2011 Aug 24]; 169 [about 38p]. Available from: http:/ /www.popcouncil.org/pdfs/wp/169.pdf

31. World Health Organization (WHO). Impact of Aids on Older People in Africa: Zimbabwe case Study [document on the Internet]. 2002. [cited 2011 Sep 5]. Available from http://whqlibdoc.who.int/hq/2002/ WHO_NMH_NPH_ALC_02.12.pdf

32. Schatz EJ. "Taking care of my own blood": Older women's relationships to their households in rural South Africa. Scand J Public Health [serial on the Internet] 2007 Aug [cited 2011 Sep 5] 35 (suppl. 69): [about 8p]. Available from: Disponível em: http:// sjp.sagepub.com/content/35/69_supp1/147.full.pdf
33. Kautz T, Bendavid E, Bhattacharya J, Miller G. AIDS and declining support for dependent elderly people in Africa: retrospective analysis using demographic and health surveys. BMJ [serial on the Internet] 2010 [cited 2011 Sep 5] 340:c2841 [about 6p]. Available from: http://www.bmj.com/highwire/filestream/ 367508/field_highwire_article_pdf/0

34. Omram AR. The Epidemiologic Transition: A Theory of the Epidemiology of Population Change. Milbank Q 2001; 83(4):731-757.

35. Schramm J M de A, Oliveira AF, Leite IC, Valente JG, Gadelha AMJ, Portela MC, Campos MR. Transição epidemiológica e o estudo de carga de doença no Brasil. Cien Saude Colet 2004; 9(4):897-908.

36. Mensah GA. Ischaemic heart disease in Africa. Heart [serial on the Internet]. 2008 [cited 2011 Aug 28]; 94 [about 8p]. Available from: http://heart.bmj. com/content $/ 94 / 7 / 836$.full

37. World Health Organization (WHO). The global burden of disease [document on the Internet]. 2004 update. 2008 [cited 2011 Sep 10]. Available from. Disponível em: http://www.who.int/healthinfo/ global_burden_disease/2004_report_update/en/ index.html

38. World Health Organization (WHO). Achieving Sustainable Health Development in the African Region: Strategic Directions for WHO 2010-2015 [document on the Internet]. 2010 [cited 2011 Sep 6]. Available from: http://www.afro.who.int/pt/component/k2/item/2345-dr-sambo-unveils-his-strategicdirections-for-who-action-in-the-african-region2010-2015.html

39. Baingana FK, Bos ER Changing patterns of disease and mortality in Sub-Saharan Africa: an overview. In: Jamison DT, Makgoba MW, editors. Disease and Mortality in Sub-Saharan Africa [book on the Internet]. 2006 [cited 2011 Aug 28]. Available from: http:/ /www.ncbi.nlm.nih.gov/books/NBK2281/

40. Charlton K E, Rose D. Nutrition among Older Adults in Africa: the Situation at the Beginning of the Millenium. J Nutr 2001; 131(9):2424S-2428S.

41. Bekibele CO, Gureje O. Self-reported visual impairment and impact on vision-related activities in an elderly Nigerian population: Report from the Ibadan Study of Ageing. Ophthalmic Epidemiol 2008; 15(4):250-256

42. Bekibele CO, Gureje O. Fall incidence in a population of elderly persons in Nigeria. Gerontol 2010; 56(3):278-283.

43. Lasisi AO, Abiona T, Gureje O. The prevalence and correlates of self-reported hearing impairment in the Ibadan Study of Ageing. Trans $R$ Soc Trop Med Hyg. [serial on the Internet]. 2010 Aug [cited 2011 Sep 8]; 104(8):[about 5p]. Available from: http:// www.ncbi.nlm.nih.gov/pmc/articles/PMC2904808/ ?tool=pubmed

44. Gureje O, Ogunniyi A, Kola L. The profile and impact of probable dementia in a sub-Saharan African community: Results from the Ibadan Study of Aging. J Psychosom Res 2006; 61(3):327-333. 
45. Clausen T, Wilson AO, Molebatsi RM, HolmboeOttesen G. Diminished mental- and physical function and lack of social support are associated with shorter survival in community dwelling older persons of Botswana. BMC Public Health [serial on the Internet]. 2007 [cited 2011 Sep 8]; 7:144 [about 8p]. Available from: http://www.ncbi.nlm.nih.gov/pmc/ articles/PMC1925080/

46. Debpuur C, Welaga P, Wak G, Hodgson A. Selfreported health and functional limitations among older people in the Kassena-Nankana District, Ghana. Glob Health Action [serial on the Internet]. 2010 [cited 2011 Sep 8]; S2 [about 10p]. Available from: http://www.ncbi.nlm.nih.gov/pmc/articles/PMC 2957305/?tool=pubmed

47. Tawfik L, Kinoti S. (2001). The impact of HIV/AIDS on the health sector in sub-Saharan Africa: the issue of humanresources. The SARA Project [document on the Internet]. 2001 [cited 2011 Sep 5]. Available from: http://www.dec.org/pdf_docs/PNACK673.pdf

48. Goudge J, Gilson L, Russell S, Gumede T, Mills A. Affordability, availability and acceptability barriers to health care for the chronically ill: Longitudinal case studies from South Africa. BMC Health Serv Res [serial on the Internet]. 2009 [cited 2011 Aug 28]; 9(75)[about 18p]. Available from: http://www. biomedcentral.com/content/pdf/1472-6963-9-75.pdf

49. African Union, HelpAge International. Policy framework and plan of action on ageing [document on the Internet]. 2003 [cited 2011 Aug 22]. Available from: http://www.helpage.org/silo/files/au-policyframework-and-plan-of-action-on-ageing-.pdf

50. Aboderin I. Understanding and Advancing the Health of Older Populations in sub-Saharan Africa: Policy Perspectives and Evidence Needs. Public Health Reviews 2010; 32(2):357-376.

Artigo apresentado em 22/07/2012

Aprovado em 28/08/2012

Versão final apresentada em 19/09/2012 